\begin{tabular}{|c|c|c|c|}
\hline $\begin{array}{l}\text { Vingtième Siècle. } \\
\text { Revue d'histoire } \\
\text { Publiée trimestrielle- } \\
\text { ment avec le concours } \\
\text { du Centre national du } \\
\text { livre et de I'lnstitut des } \\
\text { sciences humaines du } \\
\text { Centre national de la } \\
\text { recherche scientifique } \\
\text { - 2015, Presses de la } \\
\text { Fondation nationale des } \\
\text { sciences politiques } \\
\text { Conception graphique: } \\
\text { Nicolas Hubert } \\
\text { Directeur de publication: } \\
\text { Jean. Pierre Rioux }\end{array}$ & 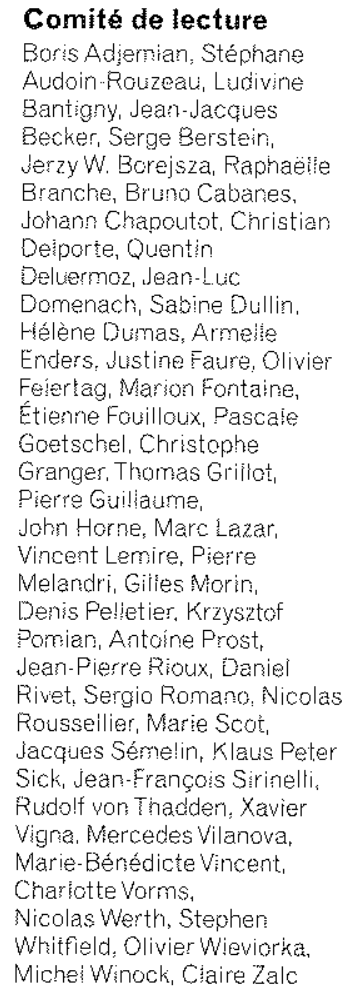 & 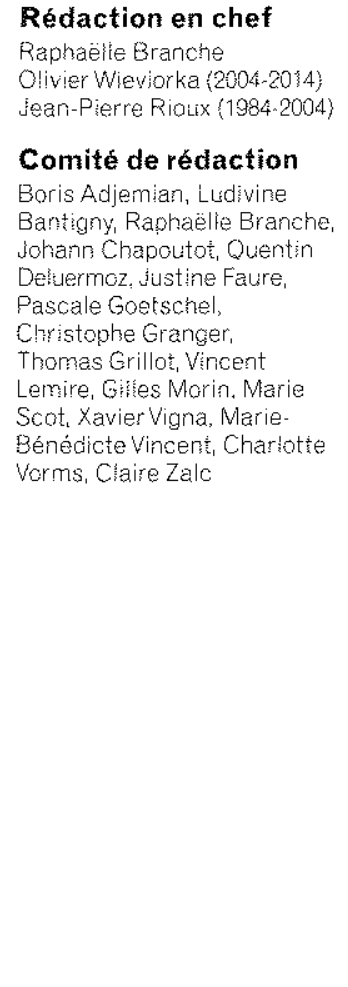 & 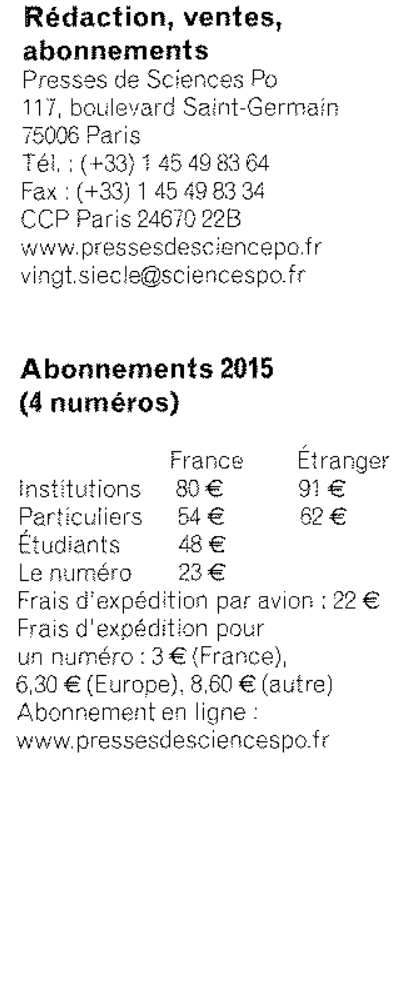 \\
\hline
\end{tabular}

\title{
Parti communiste et féminisme
}

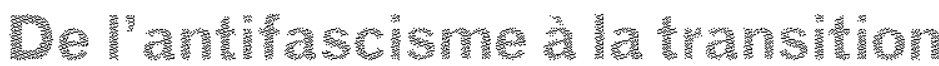 démocratique en Espagne}

Mónica Moreno-Seco

L'article de Mónica Moreno-Seco invite à analyser les relations entre féminisme et communisme espagnols à deux moments clés de leurs histoires, les années 1930 , de la République à la guerre civile, et les années 1970, à l'âge de la Transition démocratique. Il met en lumière les dynamiques croisées et les stratégies complémentaires et concurrentes entre les deux mouvements. Celles-ci évoluent entre les années 1930 et les années 1970, mais sont toujours spécifiques, par comparaison avec d'autres pays européens.

Le Parti communiste espagnol (PCE) a joué un rôle politique remarquable au cours de deux moments cruciaux du $20^{e}$ siècle espagnol : la guerre civile (1936-1939) et la Transition démocratique du franquisme à la démocratie dans les années 1970. A ces deux occasions, il s'est présenté comme un ferme défenseur de la démocratie : que ce soit au nom de l'antifas. cisme et de la République agressée par le coup d'État de 1936 ou comme force directrice de l'antifranquisme dans les années 1960 et 1970 '. À ces deux moments, le PCE s'est déclaré

(i) Jean Vigreux et Serge Wolikow insistent sur l'influence de la démocratie libérale dans les partis communistes occidentaux. Voir Cultures communistes aut $20^{\circ}$ siecle : entre guerre et modernité, Paris, La Dispute, 2003, p. 10. Sur l'évolution du Parti communiste espagnol (PCE), voir Manuel Bueno, José Hinojosa et Carme Molinero, Historia del PCE : I Congreso,
1920 1977, Madrid, FIM, 2007. partisan des droits des femmes et a adopté une stratégie d'ouverture favorisant, en période de crise et de changement, la collaboration avec les différents milieux et les différentes cultures politiques de la société espagnole.

Depuis la chute du franquisme, l'historiographie espagnole a témoigné un intérêt croissant pour la relation entre les femmes et le PCE, pour le militantisme communiste féminin et, dans une moindre mesure, pour ses liens avec le féminisme. Les premiers travaux de Giuliana Di Febo ou de Fernanda Romeu Alfaro ont démontré que le communistme avait défendu des valeurs d'égalité, mais sans les avoir toujours appliquées en pratique ${ }^{2}$. Expression de ce paradoxe, les femmes sont toujours restées minoritaires aux postes de responsabilité dont dépendaient l'organisation et la représentation du Parti. Il existe diverses interprétations de cette contradiction: celles qui insistent sur la responsabilité des femmes, lesquelles subordonnèrent les intérêts des femmes à la lutte politique au moins jusqu'à la fin de la Transition démocratique ${ }^{3}$, et celles qui soulignent au contraire le poids

(2) Giuliana Di Febo, Resistencia y movimiento de mujeres en (2paña, 1936-1976, Barcelone, Icaria, 1979 ; Fernanda Romeu Alfaro, Elsilencio roto : mujeres contra el franquismo, Madrid, édi-

(3) Maria José Valverde Márquez, «La mujer y el Partido Comunista de España (1958-1977) ", in Maria José Jiménez Tomé (dir.), Pensamiento, imagen, identidad : a la búsqueda de definición de género, Malaga, Universidad de Málaga, 1999 , 
des conceptions traditionnelles de genre dans le Parti et la méfiance, voire la misogynie, des camarades masculins ${ }^{1}$. Ce texte se rattache à la seconde interprétation, mais adopte une position nuancée. Il ne faut pas oublier en effet la pluralité des trajectoires et des conceptions des militantes. Si le Parti a effectivement instrumentalisé le travail politique des femmes, certaines d'entre elles ont parfois pu exercer une influence sur l'organisation du PCE. En défendant l'émancipation des femmes, nombre de militantes communistes, qu'elles se déclarent ou non féministes, ont introduit des principes, des ressources symboliques et des pratiques qui, à différents degrés, ont transformé la culture politique à laquelle elles appartenaient en imposant l'idée d'égalité entre les genres, et ont ainsi reformulé les identités communistes.

Cet article entend donc démontrer que l'ouverture du PCE au féminisme, dans les années 1930 comme dans les années 1970 , n'est pas le produit de la seule stratégie du PCE, mais résulte aussi de l'action des militantes communistes. Il propose à cette fin une analyse comparative de l'expérience des années 1930 et 1970, deux périodes d'effervescence politique pendant lesquelles le PCE et le féminisme ont eu une influence publique importante. On présentera une réflexion sur l'évolution des relations entre le mouvement des femmes et le communisme et on s'interrogera sur la continuité ou la rupture entre ces deux moments historiques. Bien entendu, il ne s'agit pas d'oublier qu'à d'autres époques, comme celle de l'aprèsuguerre par exemple, le PCE a soutenu des approches de genres traditionnelles. Limage des femmes au sein du Parti a beaucoup

(I) Francisco Erice Sebares, « Mujeres comunistas : la miliancia femenina en el comunismo asturiano, de los origenes al final del franquismo ", in Valentin Brugos (dir.), Los comuaistas en Asturias (1920-1982), Gijón, Trea, 1996, p. 314 Marta del Moral Vargas, Acción collectiva femenina en Madrid (909-1931), Santiago, Universidade Santiago de Compostela, 2012 , p. 309-325. changé, subordonnée qu'elle était au contexte politique et aux intérêts du Parti ${ }^{2}$. Pour autant, les relations entre les femmes et le PCE ne se déduisent pas des seuls intérêts immédiats du Parti, mais ont toujours été influencées, aussi bien dans les discours que dans les pratiques, par des éléments aussi divers que la théorie marxiste sur l'oppression des femmes, les directives de l'URSS, la stratégie de la direction du PCE, auxquelles il faut ajouter l'action des militantes communistes et les apports du féminisme lui-même.

Cette recherche s'appuie sur les archives du PCE conservées à Madrid, qui fournissent des sources pertinentes surtout pour les années 1960 et 1970 , ainsi que sur la presse communiste, sur de nombreuses brochures et sur des mémoires de militantes et de personnes en contact avec elles, rédigées ał moment des événements ou lorsque s'est ouvert le débat public des années 1990 sur la mémoire de la guerre civile et de l'époque franquiste.

\section{Eqalité, révolution et antifascisme \\ dans les années 1930}

Sous la Seconde République (1931-1936), le Parti communiste espagnol (PCE) est une petite formation politique dont le militantisme féminin est négligeable. Créé en 1921, le PCE adopte une attitude très critique vis-à-vis du nouveau régime démocratique républicain, à partir de positions ouvriéristes et révolutionnaires ${ }^{3}$. Le mouvement féministe, de son côté, se développe de manière très dynamique dans les années 1920 et contribue à l'intégration dans la Constitution républicaine de 1931 du

(2) Eric D. Weitz, «L'home heroic i la dona eterna : gènere política en el comunisme europeu, 1917-1950 $\%$, Afers, 33-34, 999, p. $393-414$

(3) Rafael Cruz, El Partido Comunista de España en la ll República, Madrid, Alianza, 1987. droit de vote des femmes et du divorce ${ }^{1}$. Entre le mouvement féministe et le $\mathrm{PCE}$, les contacts sont inexistants, notamment parce que le $\mathrm{PCE}$ considère le féminisme comme un mouvement bourgeois et inutile et parce qu'il est convaincu que l'égalité arrivera d'elle-même avec le triomphe de la révolution sociale. La situation évolue cependant rapidement.

\section{Sous la République, premier rapprochement}

Sous le régime républicain, un premier rapprochement entre féministes et femmes communistes s'opère. Le PCE tente d'utiliser l'intérêt éveillé par le processus révolutionnaire en URSS pour élargir son influence. Pour donner une image des progrès réalisés dans « la patrie du socialisme », le PCE prend pour exemple les avancées économiques soviétiques, plus rarement l'égalité entre femmes et hommes. Le nouveau régime a en effet octroyé de nombreux droits aux femmes dès 1917 (le vote, le divorce, l'avortement) et a permis le dévelopw pement d'initiatives comme le Jenotdel, l'organisation des femmes du Parti communiste d'Union soviétique, qui militait en faveur de changements dans les relations de genre. Cependant, la situation des femmes ne fait pas partie des priorités du PCE au début des années 1930. C'est pour cette raison que, au-delà de vagues allusions aux succès obtenus par les Soviétiques, on insiste d'abord sur le potentiel des femmes dans la lutte, en évoquant notamment leur participation aux journées révolutionnaires de $1917^{2}$. Les références commencent à être plus fréquentes au milieu des années 1930, précisément quand les initiatives qui modifient le plus l'ordre établi en URSS, comme le Jenotdel, sont définitivement abandonnées, et quand la propagande soviétique

(x) Ana Aguado, «Entre lo público y lo privado : sufragio y divorcio en la Segunda República », Ayer, 60, 2005, p. 105-134. (2) La Internacional Comunista, avril 1933. impose un modèle de féminité classique définie par la beauté, la famille et la maternité ${ }^{3}$. Dans la presse communiste espagnole, certaines de ces mesures sont justifiées, même si elles contredisent les arguments que le Parti défend sur le sol espagnol. Le journal Mundo Obrero motive par exemple le retour à la pénalisation de l'avortement en URSS en expliquant que ce dernier a perdu sa pertinence dans une société où les femmes jouissent déjà de l'égalité totale avec les hommes, alors que, dans les sociétés capitalistes, la lutte pour l'avortement continue d'être nécessaire

A partir de 1934, la popularité du Parti com muniste commence à grandir, tout d'abord parce que le tournant stratégique opéré par le Komintern inaugure (pour tous les partis communistes) une politique nouvelle de collaboration « antifasciste », puis parce que le PCE s'attire des sympathies à la suite des campagnes de solidarité face à la répression de l'insurrection d'octobre 1934 par le gouvernement républicain conservateur's ${ }^{s}$ En intégrant le Front populaire et en renonçant à la rhétorique révolutionnaire, le $\mathrm{PCE}$ augmente considérablement son audience, surtout avec la campagne électorale qui s'ouvre en février $1936^{\circ}$. Le Parti ouvre ses portes à la participation des classes moyennes et des intellectuels, ce qui entrâne un élargissement de la base sociale des militantes, jusqu'alors surtout d'origine ouvrière, comme Dolores Ibárruri (surnommée la « Pasionaria »). L'entrée des femmes en politique se heurte alors à des

(3) Wendy Goldman, «Les femmes dans la société sowiéique », in Michel Dreytus et al., Le Stecte des communismes, Paris, Éd. de l'Atelier/ Editions outrières, 2000, p. 187-192. (4) Mundo Obrero, 27 avril 1936, in Rafael Cruz, El Partido Comunista de España..., op. cit., p. 98 (5) Antonio Elorza et Marta Bizcarrondo, Queridos camata-
das: la Internacional Comanista y España, 1919-1939, Barcelone, Planeta, 1999, p. 238-259.

(6) Rafael Cruz, « Del partido recién llegado al partido de todos : el PCE, 1920-1939 $\gg$, in Actas del Y Congreso sobre la bistoria del PCE, 1920-1977, Oviedo, FLM, 2004 (cd-rom). 
résistances parmi les membres masculins, fermement critiquées par Ibárruri elle-même. Dans un Parti où le militantisme est majoritairement masculin, Ibárruri occupe une position de pouvoir remarquable, acquise à la suite de son élection comme députée en 1936 et qu'elle conserve pendant la guerre civile, même si son poids réel dans la prise des décisions politiques est en réalité resté en deçà de son importance publique $^{1}$. En février 1936, le PCE affirme que plus d'un tiers de ses nouveanx militants sont des femmes ${ }^{2}$. Larrivée de femmes instruites et familiarisées avec les débats féministes permet de consolider le travail de la Commission fémiw nine du PCE, que dirige Ibárruri et à laquelle collaborent des militantes comme l'institutrice Encarnación Fuyola. On assiste à l'intégration d'intellectuelles admiratives des réformes introduites en URSS, comme Maria Teresa León, qui se consacre à des tâches de propagande pro-soviétique dans des magazines culturels, ou comme Irene Falcón, qui fréquente les milieux intellectuels madrilènes et s'intéresse très tôt au féminisme ${ }^{3}$. Le PCE attire aussi de nombreuses jeunes femmes venues d'organisations étudiantes ou qui trouvent au sein du Parti communiste un espace de formation culturelle et politique ${ }^{4}$

L'existence d'espaces de sociabilité progressistes permet le contact entre femmes issues des différentes cultures politiques, pacifiste, féministe et communiste. Les forums de

(r) Rafael Cruz, Pasionaria : Dolores Ihairruri, historia y simbolo, Madrid, Biblioteca Nueva, 1999, p. 90-93 et p. 129.

(2) Selon Fernando Hernández, Sánchez, Guerra o revolución : el Purtido Comunisista de España en la guerra civil, Barcelone, Crítica, 2010, p. 72

(3) Allison Taillot, «El modelo soviético en los años $30: 10$ viajes de María Tèresa León y Ráael Alberti a Moscú $\gg$, Cabier de civilisation espagnole contemporaine, 9, 2012 ; Irene Falcón, Asalto a las cielos : mi vida junto a Pasionaria, Madrid, Temas de Hoy, 1996, p. 63-87.

(4) Carmen Parga, Antes que sea tarde, Madrid, Compañu Literaria, 1996, p. 34-35; Alejandra Soler, La vida es un $r$ o caudaloso con peligrosos rápidos: Al final de todo... sigo comunist Valence, Presses universitaires de Valence, 2009, p. 24-26. débat intellectuel comme le Lyceum Club ou la Résidence des Demoiselles à Madrid cohabitent avec les initiatives patronnées par le PCE ou proches de celui-ci. Certaines féministes connues, bien que d'affiliation républicaine ou socialiste, appartiennent ainsi à l'Association des amis de l'Union soviétique créée en 1933, qui essaie d'offrir une image positive des acquis de la Révolution, en mettant particulièrement en valeur les droits des femmes ${ }^{3}$. Le Secours rouge international, organisation satellite du PCE, fournit quant à lui des contacts avec les intellectuelles, dont certaines deviennent membres du Parti, comme Matilde Landa ${ }^{6}$. Une autre initiative très importante est le développement de la section espagnole du Comité mondial des femmes contre la guerre et le fascisme qui tient son premier congrès national au cours de 'été 1934. Dans le comité national espagnol, on trouve des personnalités comme les républicaines Victoria Kent et Clara Campoamor ou la socialiste Margarita Nelken, même si Dolores Ibárruri en détient la présidence effective $^{7}$. D'après Mercedes Yusta Rodrigo, cette association ne s'explique pas seulement par les intérêts du parti communiste, mais également par le fait qu'elle a réussi à rassembler des femmes portées par d'autres idéologies et intérêts ${ }^{8}$.

(5) María Magdalena Garrido Caballero, « Las relaciones entre España y la Unión Soniética a través de las Asociaciones de Amistad en el siglo $\mathrm{XX} \gg$, thèse de doctorat en histoire, Universidad de Murcia, 2006.

(6) Laura Branciforte, El Socorro Rojo Internacional (1923-1939): relatos de la solidaridad antifascista, Madria, Bibliotea Nueva, 2011 ; David Ginard, Matilde Landa, de la ILE, a las cárcetes franquistas, Madrid, Flor del Viento, 2005. (7) Irene Falcón, Asalto a los cielos..., op. cit., p. 98-100 Dolores Ibárruri, El uninico camino, Moscou, Progreso, 1962, 1976 , p. $184-191$

(8) Mercedes Yusta Rodrigo, « La construcción de una cultura política femenina desde el antifascismo (1934-1950)» in Ana Aguado et Teresa Maria Ortega (dir.), Feminismos y ant feminismos: culturas polititas e identidades de género en la España del sigh XX, Valence, Presses universitaires de Valence, 2011 p. 258-266.
Communisme et émancipation des fernmes pendant la guerre civile

Pendant la guerre civile (1936-1939), le PCE devient la première formation politique républicaine, tandis que le mouvement féministe perd ses organisations, qui subissent la répression franquiste et dont les dirigeantes doivent souvent fuir à l'étranger. Les revendications féministes restent néanmoins présentes dans le débat public. Si le PCE domine celuici, d'autres forces politiques, notamment les socialistes et les anarchistes, s'en emparent. En outre, le recrutement féminin au PCE, commencé avant 1936, s'accélère avec la guerre. Les hommes étant appelés aux armes, le Parti compte sur les femmes pour garantir son fonctionnement et soutenir l'effort de guerre à l'arrière ${ }^{1}$. Le discours insiste désormais sur l'appel aux nouvelles militantes et sur la promotion interne des femmes2. D'après Fernando Hernández Sánchez, en mars 1937, le PCE déclare 245532 membres, dont 18329 femme $(7,5 \%$ des effectifs). Après avoir atteint son apogée à la fin de l'année avec trois cent quarante mille militants, le PCE connât une baisse générale de ses effectifs, mais enregistre au contraire une augmentation du nombre de ses militantes, aboutissant à une féminisation du Parti, avec des taux qui atteignent, en $1938,30,5 \%$ des effectifs à Madrid et $19,5 \%$ à Barcelone. Même si ces chiffres reflètent surtout la situation des grandes villes à la fin de la guerre civile, la féminisation est réelle et rencontre d'ailleurs des résistances parmi les camarades masculins 3 . La diversité des origines s'accroît avec l'arrivée d'intellectuelles $-\infty-$

(i) Sur l'expérience des femmes dans le conflit, voir Mary Nash, Rojas: las mujeres repubticanas en la Guterra Civil, Madrid, Taurus, 1999

(2) Resolution du Comité central du PCE dans La Internacional Comunista, ${ }^{\text {ter juillet } 1938 .}$

(3) Fernando Hernandez Sánchéz, Guerra o pevolución. op. cit., p. 286-293. Lauteur souligne la fragilité des statistiques du PCE soumises à des impératifs de propagande, confrontées comme Margarita Nelken, ancienne députée socialiste et féministe renommée, de bourgeoises, comme Constancia de la Mora ou Caridad Mercader, conduites au communisme par leurs préoccupations sociales, d'ins titutrices, comme Dolors Piera, et surtout d jeunes ouvrières et étudiantes comme Teres Pàmies ${ }^{4}$.

L'augmentation des effectifs féminins du Parti s'accompagne-t-elle de l'introduction des thèses féministes? À cette même époque la référence aux tâches maternelles et domestiques considérées comme spécifiquemen féminines se renforce au sein du communisme international, aussi bien en URSS que dans les partis d'Europe occidentale. Or la guerre en Espagne donne une image différente des femmes, actives et combattantes, s'incarnant notamment dans la figure de la milicienne. Dans les partis communistes soviétique et occidentaux, cette situation est interprétée comme une réponse exceptionnelle à une situation exceptionnelle, qui ne fait, d'après Brigitte Studer, que confirmer la norme dominante ${ }^{5}$. Il est vrai que l'engagement armé des femmes reste un phénomène marginal et que même les militantes communistes ont peu en commun avec l'expérience des miliciennes. Mais elles ne luttent pas moins pour que les femmes puissent exercer une activité de résistance à l'arrière, du fait de leurs contacts avec les féministes et de leur désir de défendre la République qui a accordé de nombreux droits aux femmes.

à des évolutions rapides de sa composition et contenant de nombreuses exreurs.

(4) Luis Mercader (fils de Caridad), Ramnón Mercader, mi bermano, Madrid, Espasa-Calpe, 1990, p. 38-46. ll existe aussi d'intéressantes biographies. Voir notamment Soledad Fox, Constancia de la Mora. Esplendor y sombra de una wida española del siglo XX, Salamanca, Espuela de Plata, 2008; Josebe Martínez Gutiérrez, Margarital Nelken (1986-1968), Madrid, Del Orto, 1997.

(5) Brigitte Studer, \& La femme nouvelle », in Michel Dreyfus et al., Le Siède des communitsmes, Paris, Éd.de l'Atelier' Éditions ouvrières, 2000 , p. 383-385. 
Cette particularité espagnole se retrouve dans les discours et les écrits de Dolores Ibárruri. Pendant la campagne électorale de février 1936, celle-ci s'adressait déjà aux femmes en tant que mères, tout en identifiant le fascisme à une idéologie oppressive à l'égard des femmes, sur la base d'arguments féministes ${ }^{2}$. Pendant la guerre civile, celle-ci condamne toujours la violence faite aux femmes et aux enfants victimes du fascisme et en appelle à l'effort des mères résistantes. Mais ce registre victimiste et maternaliste coexiste encore une fois avec un registre féministe. À l'occasion de la journée internationale de la femme le 8 mars 1938 , elle affirme par exemple qu'il faut « cesser de considérer la femme comme une citoyenne de catégorie inférieure et lui accorder la plénitude de droit qu'elle mérite $\gg$. Elle propose, après l'inévitable référence aux femmes soviétiques, de ne parler de « démocratie et d'émancipation que lorsque les femmes auront atteint l'indépendance économique ${ }^{2}$ ». De même, dans son intervention à la Conférence provinciale du Parti à Madrid tenue en 1938, elle s'adresse ainsi à ses camarades:

« Camarades, un homme ne peut pas se dire révolutionnaire et avoir une conception aussi réactionnaire du rôle dévolu à la femme. La femm ne doit pas être la femelle avec laquelle il couche. Elle ne doit pas être la femme qui lui recoud ses chaussettes et lui prépare le pot-au-feu à l'heure; la femme doit être la compagne, elle doit être camarade avec qui partager les peines, la fatigue, les désagréments et aussi les joies de la lutte ${ }^{3}$.

Elle tient là un discours qui unit émancipation et communisme, et qui ne subordonne pas les droits des femmes à la victoire ou aux

(I) Mercedes Yusta Rodrigo, « La construcción de un cultura politica femenina... », op. cit., p. 268.

(2) Pasionaria, iA las mujeres madrileñas!', Madrid, Partido

(3) Mundo Obrero, 7 janvier 1938. intérêts du Parti, mais qui conçoit les deux comme inséparables, introduisant ainsi des éléments nouveaux dans la culture politique communiste espagnole.

Sans nier l'existence d'autres voix, le discours de Dolores Ibárruri symbolise tout à fait les contradictions du Parti communiste. Mais il montre aussi comment celle-ci a su utiliser différents registres afin de mobiliser les Espagnoles pour la défense immédiate de la République et pour l'édification d'une société plus juste après la victoire. Son image atteint une popularité particulière pendant la guerre : de par ses origines humbles, sa tenue et sa coiffure traditionnelles, elle incarne la femme espagnole; mais de par son rôle de dirigeante politique et ses capacités d'oratrice, elle incarne un Parti qui défend l'égalité. Cette combinaison fait d'elle un mythe ${ }^{4}$. Il existe cependant deux interprétations divergentes de son discours : certains, comme Maria José Capellín ou Maria Carmen García Nieto, soulignent son apologie du rôle des femmes dans la société et dans le Parti ; d'autres, comme Yannick Ripa, rappellent son acceptation du discours traditionnel dans lequel l'émancipation féminine ne bénéficie d'aucun traitement spécifique ${ }^{5}$. Ces deux interprétations éclairent chacune un aspect particulier : on peut affirmer qu'Tbárruri a défendu des principes féministes, nuancés par des appels au maternalisme, atteignant ainsi un difficile équilibre entre égalité et complémentarité. Faire accepter aux femmes communistes le qualificatif de féminisme était par ailleurs très difficile, car le terme était toujours

(4) David Ginard, «"La madre de todos los camaradas" : Dolores Ibárruri como símbolo movilizador, de la Guerra Civil a la transición posfranquista », Ayer, 90, 2013, p. 189-216. (5) Maria José Capellín Corrada, De la casa al compromiso polititcon: Dolores lbarruri, mito del pteblo, 1916-1939, Madrid, Fundación Dolores baatruri, 1996; Maria Carmen Garcia Nieto, « Hija de una época y de una clase, mujer con las mujeres : Dolores Ibárruri », Arenal, 3 (2), 1996, p. 259-277, Yannick Ripa, «Le mythe de Dolores Ibátruri », Chio, 5, 1997, p. $147-155$ associé à un mouvement bourgeois divisant les femmes et les hommes. Mais bien qu'elles n'en aient pas accepté le nom, on peut considére leurs propositions et leurs initiatives comme féministes ${ }^{1}$.

L'activité politique et sociale des femmes communistes prend place notamment au sein de l'Asociación de Mujeres Antifascista (AMA, Association des fermmes antifascistes), héritière du Comité des femmes contre la guerre et le fascisme. Cette organisation joue un rôle capital dans la mobilisation des femmes à l'arrière et, selon une de ses dirigeantes, la communiste Encarnación Fuyola, l'AMA lut tait pour les libertés démocratiques et pour les droits des femmes ${ }^{2}$. Les cercles intellectuels déjà actifs sous la République sont également sensibles aux positions des communistes ou des sympathisantes du Parti sur l'émancipation des femmes, comme l'Association d'amitié avec l'Union soviétique, très active pendant la guerre, et l'Alliance des intellectuels antifascistes, où se fait remarquer l'écrivaine Maria Teresa León.

La débâcle du camp républicain en avril 1939 entraine dans son sillage une profonde fracture politique et sociale, due à la répression systématique exercée par la dictature franquiste contre les forces politiques et syndicales de gauche (et done contre le PCE), mais aussi contre des mouvements qui contredisent les valeurs national-catholiques imposées par le franquisme, comme le féminisme. Le mouvement disparaît de manière brutale et ne réapparaîtra que dans les années 1970 à partir de sources et dans un contexte très différents. Les militantes communistes sont quant

(I) Selon Ángela Cenarro, « Antifascismo y republicanas durante la guetra civil », in Mary $\mathrm{Nash}$ (dir.), Cindadanus y protagomistas butomicas : mutjeres repubticanas en a ll Repiblica y la Guema Civil, Madrid, Congreso de los Diputados, 2009, p. 164-186.

(z) Encarnación Fuyola, Mujeres Antifascistas: su trabajo y sut organizacion, Valence, Ediciones de las mujeres antifascistas, 1936 à elles emprisonnées, obligées de s'exiler, ou contraintes de continuer leur engagement politique dans la clandestinité. Au sein du PCE, lutte pour les droits des femmes n'est plus une priorité et s'efface devant la lutte contre la dic tature ${ }^{3}$.

\section{Le "Parti de la libération de la femme" (années 1960 et 1970)}

Les années 1970 constituent un second moment où PCE et mouvement féministe reprennent de l'importance dans l'arène politique. Pendant les dernières années du franquisme, le PCE devient un pilier fondamental dans la reconstruction du mouvement des femmes et va jusqu’à se définir comme un parti féministe.

\section{Une nouvelle culture communiste}

Des années 1960 au début des années 1970, une nouvelle culture communiste s'ouvre au féminisme. Ce changement a été rendu possible par la nouvelle stratégie du PCE à partir de la fin des années 1950 : centrée sur la reconstruction d'une société civile en vue de l'implantation d'une future démocratie, elle donne la priorité au rapprochement avec les mouvements sociaux, y compris ceux qui défendent les droits des femmes. Ce processus a contribué à créer une nouvelle culture communiste davantage ouverte au changement des relations de genre ${ }^{4}$.

(3) Parmi différents travaux, voir Claudia Cabrero Blanco, "Militancia, resistencia y solidaridad: las mujeres comunistas y la lucha clandestina del primer franquismo $»$, in Manuel ria, identidad e bistoria social, Madrid, FIM, 2010, p. 205-229; rene Abad Buil, En socall, Madnd, Him, 2010, p. 205-229, 2012 ; Mercedes Gusta, Modres Coraje contra Franco : I Unión o Mujeres Fedes husta, Madres Coraje contra Franco : la Unión (1941-1950), Madrid, Cátedra, 2009.

(4) Carme Molinero, « Uma gran apuesta : la oposición política a través de la movilización social » et Francisco Errice Sabares, « El "orgullo de sex comunista" : imagen, autopercepción, memoria e identidad colectiva de los comunistas españoles », in Manuel Bueno et Sergio Gálvez (dir.), Nosotros, los comunistus..., op. cit., p. 255-283 et p. 139-183. 
Dans ce nouveau contexte, l'action politique des femmes prend un nouvel élan. Les militantes adultes continuent à défendre les prisonniers politiques et à soutenir les mobilisations ouvrières de leurs maris. Mais en parallèle, elles participent elles-mêmes à des mouvement sociaux et commencent à prendre conscience des discriminations dont elles sont victimes Cette évolution coincide avec le développement des mouvements protestataires de jeune dans les pays occidentaux au cours des années 1960 et avec l'irnuption de la seconde vague de féminisme. L'ouverture du milieu communiste au féminisme correspond aussi à l'arri vée d'une génération de jeunes militantes, étudiantes ou salariées. Ces militantes se forgent une conscience féministe par leurs lecture et par leurs contacts avec le mouvement des femmes, mais aussi en faisant l'expérience personnelle de la discrimination dans leur activit professionnelle comme dans leur activité politique. En remettant en question les relations de genre dans le Parti, des jeunes et quelque adultes contribuent à reformuler les identités communistes ${ }^{2}$. L'expérience des années 1930 n'est pas une référence pour ces jeunes féministes, parce que les militantes les plus combatives pendant la guerre civile ont disparu ou sont en exil. La majorité des communistes adultes soutiennent des positions modérées sur a question des femmes.

La documentation interne du Parti témoign du fait que le féminisme est désormais considéré comme indispensable pour aller vers l'égaité pendant et après la révolution. Révolution sociale et égalité entre femmes et hommes

(I) Irene Abad Buil, « Reivindicaciones y movilizaciones emeninas desde el PCF durante el Segundo francuismo $»$ in ibid, p. 231-252.

(2) Mónica Moreno-Seco, « A la sombra de "Pasionaria" : mujeres y militancia feminista (1960-1982) , in Maria Dolore Ramos (dir.), Tejedoras de ciudadania : culturas politicicas, femin mos y luchas democraticas en Eispana, Malaga, Universidad de Málaga, 2014, p. 257-282. cessent d'être considérées comme des projets opposés : un rapport interne du PCE du début des années 1970 affirme ainsi que les femmes ont certes besoin du socialisme pour leur libération, mais que la révolution a aussi besoin des femmes ${ }^{3}$. Comme les revendications féministes s'inscrivent dans une lutte pour la démocratie, elles constituent elles aussi une voie vers le socialisme. Les puvres de Simone de Beauvoir ou de Betty Friedan viennent s'ajouter aux textes classiques du marxisme pour forner une base théorique qui intègre déjà les apports du suffragisme. Parallèlement à cette moture avec les positions classiques du PCE, le modèle soviétique perd de son rayonnement, à l'heure du ralliement du PCE à l'eurocommuisme, et on affirme même que, dans les pays socialistes, les femmes n'ont pas obtenu l'égalité complète. Lidée de réunir les femmes de toutes origines sociales dans des associations féministes gagne du terrain.

C'est dans ce contexte évolutif que s'inscrivent les propositions théoriques de Giuli Adinolfi, militante formée en Italie qui, au milieu des années 1960, promeut la création d'un groupe de réflexion avec d'autres communistes à Barcelone pour étudier des textes féministes. Dans la revue clandestine Nous Horitzons, publiée par des intellectuels communistes, Adinolfi critique les thèses qui conçoivent l'activisme des femmes comme une tion subalterne sans objectifs spécifiques ou qui soutiennent que la révolution éliminera de manière automatique la discrimination et, par conséquent, que les femmes ne doivent pas développer de militantisme spécifique. Elle rejette aussi la conception « féministe » (elle ne se qualifie pas encore ainsi) qui considère

3) Archive historique du PCE (AHPCE), Organizaciones de Mujeres (OM), bote 11 , chemise $12 / 1$, « Sobre la mujer oaquin, s. d. Ces rapports ne sont pas signés or sont signes vec un pseudonyme en raison des règles imposées par la clandestinitế. que l'accès au travail et à l'éducation règlera à hui seul les problèmes des femmes, alors qu'en réalité, affirme-t-elle, l'origine de la discrimination réside dans les structures de la sociét et dans l'exploitation. Adinolfi conclut en sou lignant que les femmes, par leur action, ont un rôle propre à jover dans la construction d'un nouveau pays. Ces idées sont reprises par le Parti, qui propose de créer un mouvement de femmes qui unirait la lutte pour l'émancipation des femmes à la lutte pour la justice sociale ${ }^{1}$ Les thèses d'Adinolfi constituent le fondement théorique du Movimiento Democrático de Mujeres (MDM, Mouvement démocratique des femmes)

En pleine clandestinité, l'idée resurgit de développer une organisation ouverte aux femmes d'origines et d'idéologies diverses, sous la direction de militantes communistes. Principale organisation dans la reconstruction d'un mouvement féministe en Espagne, le MDMI est fondé en 1965 et est longtemps dirigé par des militantes communistes comme Dulcinea Bellido et Mercedes Comabella. Le MDM donne la possibilité au PCE d'être présent au sein du mouvement et de s'imprégnet de la théorie féministe. A partir de question ancrées dans le quotidien, comme les prix à la consommation, les services urbains de base ou l'éducation des enfants, le MDM introduit de manière progressive des thèses féministes, ce qui conduit à une relecture du marxisme et à une redéfinition de ses activités ou objectifs.

(r) Lhuisa Vives (pseudonyme d'Adinolfi), « Por un planteamiento democrático de la lucha de las mujeres ", Nous Horitzons, 12, 1967, p. 30-34. Ses propositions apparaissent dans un document interne du PCE : AHPCE, OM, boite 117 chernise 12/1, «El trabajo político entre las mujeres... », s. d. Voir aussi Pilar Fibla et Camne Vilaginés, "Giulia Adinolfi, trujer y ciudadana ejemplar $"$, Mientras Tanto, 94, 2005, p. $25-30$.

(2) Irene Abad Buil, « Movimuento Democrático de Mujeres : unt vehiculo para la búsqqueda de una nueva ciudadanía lemenina en la transicion española $»$, in Actes del Congres \& $L a$

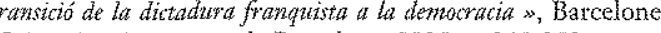
Universitat Autònoma de Batcelona, 2005, p. 245-252.
Les relations entre PCE et MDM ont éte diversement interprétées par les féministes certaines soulignent la manipulation du mouvement par le Parti', tandis que d'autre mettent l'accent sur son indépendance. C'est le cas notamment des militantes communistes, qui, comme Rosalía Sender, déclarent: « C'est nous les communistes féministes qui l'avon développé en premier mais pas d'après le consignes du Parti, au contraire, souvent contre l'opinion des dirigeants ${ }^{4}$, $\gg$ L'historiographi converge sur la survellance exercée par le parti sur le MDM et sur la division des militantes communistes à propos de l'orientation féministe et de l'autonomie du MDM par rapport au Parti ${ }^{5}$. La documentation interne du PCE, quant à elle, révèle certes l'hégémonie des femmes communistes dans le $\mathrm{MDM}^{6}$, mais confirme aussi les tensions entre les communistes du MDM et les camarades qui ne comprennent pas leurs propositions et considèrent le travail féministe comme contingent ${ }^{7}$.

La culture communiste telle qu'elle a existé sous le franquisme, et notamment sa conception inégale des rapports entre les sexes, entre en crise à cette époque, remise en question

(3) Liđia Falcón, Mujer y poder politico, Madrid, Vindicación Feninista, 1992, p. 206

(4) Rosibs Senter Begue

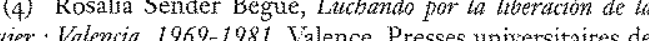
Valence, 2006, p. 111 et p. 160

(5) Vicenta Verdugo Martí « El Movimiento Democrítico de Mujeres : el compromiso político por una ciudadaní democrática », in Ana Aguado et Luz Sanfelin (dir) Comino de democracia : ciuddadanias y culturas democráticas en el sigto XX Grenade, Comares, 2014, p. 115-131; Francisco Arriero Sanz, « El Movimiento Dernocrático de Mujeres: del antifrancuismo a la movilización vecinal y fenunista », Historta Trabujo y Sociedad, 2, 2011, p. 33-62.

(6) AHIPCE, OM, boite 117 , chemise $2 / 3$, rapport sur les Journées de la libération de la femme de décembre 1975 , s. d. rapport de Amaya « Reunión de las Mujeres Demócratas en Madrid ", reçu le 28 mars 1970.

(7) ALPCE, OM, boite 117, chemise 2/3, « Reunión Nacional de Mujeres », copie du 6 juillet 1971; « Sobre el Movimiento de Mujeres Demócratas de Zaragoza », copie du 10 décembre 1971 ; « Reunión del Comité Ejecutivo del PCE con camaradas del MDM $»$, s. d. [milieu de l'année 1970]. 
par des éléments nouveaux qui interpellent aussi bien les hommes que les femmes ${ }^{1}$. À l'intérieur même du PCE, le débat éclate entre, d'un côté, des militantes qui accordent la priorité à la lutte politique sur la lutte féministe et qui pensent que la sexualité ou les droits reprom ductifs sont peu urgents, voire frivoles, et, de l'autre, des militantes plus jeunes et plus formées qui affirment que les deux combats sont parallèles et qu'il faut soutenir toutes les revendications féministes, y compris celles relatives au corps.

Le Parti connaît une évolution profonde. Le huittième congrès, qui a lieu en 1972, constitue un des moments les plus mémorables de ce processus. Santiago Carrillo y énonce la nécessité de réaliser « un virage à cent quatre-vingt degrés $\gg$ sur la question des femmes. Le changement est remarquable, au point que, lors de la « Conférence des partis communistes des pays capitalistes d'Europe sur la condition féminine » à Rome en 1974, la délégation espagnole affirme, contre l'opinion de la majorité des partis qui y assistent, que le féminisme est une lutte spécifique et que le socialisme n'implique pas automatiquement la libération des femmes. La délégation soutient ainsi des thèses très avancées par rapport à la culture communiste européenne du moment ${ }^{2}$.

À partir de ce moment-là, le PCE s'identifie de manière explicite avec le féminisme. La deuxième conférence nationale du Parti de 1975 conclut par exemple : «Nos objectifs coüncident avec ses objectifs [ceux du mouvement des fernmes] : atteindre l'égalité de la femme et de l'homme. Dans ce sens, nous sommes, nous devons être, un parti féministe. Nous sommes

(r) Xavier Domènech Sampere, « Cenizas que ardían todavía ; la identidad comunista en el tardofranquismo y la transición », in Manuel Bueno et Sergio Gálvez (dir.), Nosotros, los comutinistas..., op. cit., p. 126-132.

(2) El feminisme al PSUC : els anys setenta i vuitanta del segle $X X$, p. 35, http://elfeminismealpsuc.adpc.cat/quisom.html. le parti de la libération de la femme $»^{3}$. Cette ssimilation contribue à la reformulation de Jidentité communiste : on préconise ainsi des changements à l'intérieur du Parti, face au constat «qu'encore beaucoup de communistes ont des idées réactionnaires sur le problème féminin ». Toutefois, pour le PCE, le féminisme dit « radical » (c'est une nuance très significative) se trompe en considérant la question des femmes de manière exclusive sans prendre en considération les autres transformations politiques et sociales ${ }^{4}$.

\section{Collaboration et tensions}

entre PCE et féminisme

Au cours de la Transition démocratique (1975-1982), le PCE connaît une nette évolution idéologique, avec l'intégration totale de l'eurocommunisme 5 . Dans ce contexte, le discours officiel du Parti admet que l'arrivée de la démocratie ou du socialisme n'est pas suffisante pour régler la question de l'égalité entre les femmes et les hommes. Malgré le prestige dont jouit le PCE grâce au travail réalisé dans la clandestinité et malgré ses importants efforts de modernisation théorique, l'influence du PCE diminue parce que la démocratie ouvre de nouvelles voies de participation politique aux milieux les plus progressistes de a société qui, à maintes occasions, se distancient du Parti ou l'abandonnent. Ce processus s'observe clairement dans les relations entre communisme et féminisme. Le mouvement féministe se structure en effet sur un clivage identitaire, en s'adressant spécifiquement aux femmes, mais il a en même temps montré des préoccupations politiques inhabituelles dans

(3) Mundo Obrero, $4^{\circ}$ sem. de sept. 1975 (4)

Obrero, $4^{\circ}$ sem. de sept. 1975 et $3^{\circ}$ sem. d'oct. (5) Juan Antonio Andrade Blanco, El PCE y el PSOE en (la) transición: la evolutción ideológica de la izquierda durrante el proceso de cambio politico, Madrid, Siglo XXI, 2012. les mouvements féministes des autres pays européens, à cause du contexte de la Transition démocratique ${ }^{1}$

En tant que membres du MDM, de nombreuses communistes participent aux premières Journées de la libération de la femme a Madrid qui se tiennent au début du mois de décembre 1975, peu après la mort de Franco, et constituent le moment fondateur du féminisme de la seconde vague en Espagne. Ces Journées aboutissent à une résolution qui met en rapport la lutte des femmes avec la lutte pour la démocratie. Comme on le lit dans un rapport du PCE, cette résolution est adoptée sous l'influence des communistes, qui s'opposent à d'autres participantes lors d'intenses débats. II s'agit de déterminer « si notre lutte est une lutte contre l'homme ou une lutte contre les structures qui contribuent à la perpétuation de cette situation et, concrètement de nos jours, une lutte pour l'amnistie et les libertés ${ }^{2} \gg$. Bien que le $\mathrm{PCE}$ se considère comme féministe, les divergences avec le féminisme radical sont manifestes.

En mai 1976, trois mille femmes célèbrent les Journées catalanes de la femme à Barcelone, révélant ainsi le dynamisme croissant du féminisme espagnol. À nouveau, les femmes communistes participent, mais leurs positions ne sont plus majoritaires. Dans un bilan interne, Maria Dolores Calvet reconnait les fortes carences théoriques de beaucoup de participantes communistes. Pour elles, ces Journées agissent comme un coup de fouet qui stimule la prise de conscience de leur discrimination ${ }^{3}$.

(I) Mary Nash, «La construcción de una cultura política desde la legitimidad feminista durante la Transición politica democrática », in Ana Aguado et Teresa Maria Ortega (dir.), Ferninismos $y$ antifemintismos..., op. cit., p. 283-306.

(2) ALPCE, OM, boite 117 , chemise $2 / 3$, rapport sur les Journées, s. d. ; AHPCE, Nacionalidades, boite 75, chemise $1 / 1$, rapport de Ana, s. $d$.

(3) El femmime al PsUC..., op. cit., p. 42-53. Voir Giaime Pala, «Entre paternalismo e igualitarismo : el PSUC $y$ la cuestón de la nujer en los años del tardofranquismo », Mientras
Dans le mouvement féministe espagnol, une grande controverse éclate entre féminisme socialiste et féminisme radical : les communistes, liées au premier, soutiennent qu'à Ia lutte pour la libération de la femme il faut joindre la lutte pour la démocratie; les radi cales accusent au contraire les partis d'essayer de manipuler le mouvement. Leurs différences n'empêchent pourtant pas ces tendances de collaborer lors des campagnes pour les droits des femmes ${ }^{4}$. Ces débats et remises en question ont des répercussions à la fois dans le $\mathrm{PCE}$ et dans le MDM. Ce dernier donne la priorité à des revendications nouvelles en son sein, comme le divorce ou l'avortement', mais le mouvement entre en crise, concurrencé par d'autres organisations et accusé d'être instrumentalisé par le PCE. La présence des militantes du PCE lors des deuxièmes Journées de la libération de la femme, tenues à Grenade en 1979 , n'est plus aussi marquée.

Au même moment, les thèses féministe gagnent du terrain dans le discours officiel du Parti, ce dont témoigne la création en mai 1976 de la Commission pour les affaires féminines auprès du Comité central, qui réunit entre autres Dulcinea Bellido, Mercedes Comabella, Manuel Azcárate et Rosalía Sender. Une de ses premières initiatives est de convoquer la première conférence du Parti pour la libération de la femme, au cours de laquelle il est affirmé que cette libération est indissociable de la lutte pour la liberté, la démocratie et le socialisme. La Commission exige, peu après, la fin des discriminations légales et l'ouverture d'un débat qui surmonte les tabous sur la

Tanto, 97, 2005, p. 133-139; Soledad Bengoechea Echaondo, Les dones del PSUC, Barcelone, Els arbres de Farenheith, 2013 (4) Mercedes Augustín Puerta, Feminismo : identidad personal y luchat colectiva. Análisis del movimiento feminista español en los años 1975 a 1985, Grenade, Universidad de Granada, 2003. (5) AHPCE, OM, boite 117, chemise 2/2, progtamme de MDM-MLM, 1976. 
sexualité ${ }^{1}$ Le contact avec différents courants du féminisme permet aux secteurs les plus avancés du Parti d'approfondir les positions défendues et d'exiger « une intense révolution culturelle qui transforme la relation hommefemme $^{2}$ » pour reprendre les termes de Manuel Azcárate. Natalia Calamai plaide, quant à elle, pour séparer la sexualité de la procréation et redéfinir une nouvelle morale, éloignée de la morale bourgeoise ${ }^{3}$. Au neuvième congrès du PCE, en avril 1978, ces arguments sont intégrés dans la doctrine officielle du Parti, avec des revendications nouvelles comme la coéducation, les contraceptifs, l'avortement et « les droits au choix sexuel ${ }^{4}$.

La deuxième conférence du PCE sur la question des femmes de décembre 1978 propose de s'attaquer au problème de l'insertion des femmes dans le Parti en favorisant un nécessaire changement de mentalité dans la formation politique. L'autocritique officielle récurrente et l'inquiétude sincère des féministes du Parti n'empêchent pas le militantisme féminin et l'accession des femmes à des postes à responsabilités de rester des pratiques minoritaires. En 1977, on compte à peine $10 \%$ de femmes dans le Parti, contre $30 \%$ trois an plus tard. Pourtant, au sein de son Comité exéw cutif, les femmes, déjà peu présentes $(6,6 \%$ des membres en 1977), ont totalement dis paru en $1981^{\prime}$. Pendant les campagnes élec torales de 1977 à 1982, le PCE revendique une pleine participation des femmes à la vie publique et se présente comme le Parti «le plus féministe $\gg$. Pourtant, sur les quarante-sept

(1) Mundo Obrero, 24 novembre 1976, 1.er décembre 1976 et 15-21 décembre 1977; Hacia la liberación de la mujer : 1 Conferencia del PCE sobre la condición fernenina, Madrid, PCE 1976.

(2) Nuestra Bandera, mars-avril 1977

(3) Nuestra Bandera, juillet-août 1977

(4) Nuestra Bandera, mai-juin 1978.

(5) Nuestra Bandera, janvier 1979; Mundo Otrero, 23 février 1979 ; Mónica Moreno-Seco, «Ala sombra de "Pasionaria"... op. cit., p. 278-279. députés communistes, seules cinq sont des emmes. Le groupe parlementaire communiste propose néanmoins des projets de loi sur e divorce et sur l'avortement et présente des amendements à la Constitution pour défendre le divorce, la dépénalisation de l'avortement, des méthodes de planification familiale et la coéducation. Le PCE participe aussi aux principales campagnes féministes de l'époque ${ }^{6}$. Pourtant, à partir de 1978, l'intérêt du PCE pour le sujet décline, sous l'effet combiné de la grave crise interne qu'il traverse et de la redéfinition du féminisme à l'issue d'un conflit entre partisanes de l'égalité et partisanes de la différence.

Les années 1930 et les années 1970 sont des moments intéressants pour étudier les relations complexes du Parti communiste avec le mouvement féministe, parce qu'll s'agit de deux époques de fort rayonnement du communisme, pendant lesquelles le féminisme a en même temps constitué un enjeu du débat politique.

Ces deux moments présentent des points communs. Dans les deux cas, l'origine du rapprochement entre communisme et féminisme obéit à des préoccupations stratégiques : l'objectif est d'ouvrir le Parti aux différents milieux sociaux pour renforcer sa place dans la société. Dans les deux cas, la question des femmes parvient cependant à s'autonomiser, sous l'influence notamment des militantes communistes. Ces moments présentent auss des différences. Tout d'abord, la référence à l'URSS, importante dans les années 1930 , s'efface dans les années 1970. Ensuite, le PCE se définit en 1975 comme «le Parti de la libé ration des femmes », alors que, pendant la 1979, 21 et 23 février 1979, 10-16 septembre 1982; AHPCE OM, boîte 117 , chemise 12/3, tract « Para que no se encoja tu vida no te quedes parada : vota PCE: guerre civile, le féminisme avait été considéré comme un mouvement bourgeois. Cependant, c'est bien la guerre civile qui, en polarisant les camps, a assuré au PCE une position dominante sur la question des femmes. Au contraire, si le Parti a maintenu son influence dans la clandestinité, la question des femmes s'ouvre à d'autres organisations pendant la transition démocratique. Enfin, in ne faut pas oublier que le féminisme a également évolué : dans les années 1930 , le mouvement exigeait l'égalité formelle, que le PCE a pu reprendre à son compte pendant la guerre civile; dans les années 1970, le féminisme met davantage en avant des revendications concernant le corps et la sexualité, que le PCE finit par intégrer mais en perdant l'initiative dans le mouvement féministe.

La question de la continuité entre ces deux moments se pose également. Cette continuité existe certes dans la trajectoire de quelques militantes et dans le souvenir mythifié de la résistance politique durant la guerre civile, incarné par Dolores Ibárruri à son retour d'exil. Mais ces militantes sont peu connues dans les années 1970 et la mythification d'lbárruri se fait sans référence à ses revendications féministes des années 1930. En fait, les principales dirigeantes ont durement subi la répression franquiste et le Parti a pendant plus de vingt ans donné la priorité à l'action clandestine, remettant à phus tard la question des revendications féministes. Cela a contraint les femmes communistes à reconstruire une culture féministe ex nibilo au sein de la culture politique du PCE des années 1970

Le cas du PCE gagne enfin à être replacé dans un contexte européen. Ici, on ne peut que proposer quelques pistes, qui devraient faire l'objet de comparaisons futures. La guerre civile et la résistance à la longue dictature franquiste expliquent que l'union des forces progressistes ait été en Espagne plus qu'ailleurs érigée en valeur fondamentale par le Parti communiste, ce qui a favorisé un rapprochement entre communisme et féminisme. Parallèlement aux intérêts du PCE, l'engage ment de certaines militantes communistes permis l'ouverture au féminisme et son assimilation par la culture politique communiste espagnole. Inversement, la politisation croissante du débat public autour de l'antifascisme et de l'antifranquisme a influencé le mouvement féministe, ce qui peut expliquer que les contacts, même s'ils n'ont pas toujours été faciles, aient été maintenus avec le PCE. Cela distingue le PCE de son homologue portugais, avec lequel il partage l'expérience de la dictature mais non de l'eurocommunisme, et qui n'a jamais réussi à intégrer le féminisme et la dimension de genre à la lutte pour la démocratie ${ }^{\prime}$ Cela le rapproche en revanche du Parti communiste italien (PCI) : pendant la Seconde Guerre mondiale, celui-ci a soutenu l'engagement des fernmes dans l'antifascisme et après la guerre, est revenu à des positions traditionnelles. Dans les années 1970 , le PCI se place à la pointe du mouvement eurocommuniste, il déclare l'union entre féminisme et socialisme lors de son congrès de $1979^{2}$. En France, ces débats n'ont touché le PCF que tardivement ${ }^{3}$. Ni la participation au Front populaire, ni la présence des femmes dans la Résistance, ni le poids du Parti dans la vie politique après 1945 n'ont conduit ce dernier à changer ses positions marxistes orthodoxes. Dans les années 1970, sa présence est moins forte que celle du PCI et du $\mathrm{PCE}$ dans leurs arènes politiques respectives,

(i) Selon Manuela Tavares, Fentuinismos, percursos e desafios (1947-2007), Lisbonne, Texto Editores, 2011

(2) Karen Beckwith, « Ferninist and Leftist Politics in Italy: The Case of UDI.PCI Relations $»$, in Sylvia Bashenkin (dir), Women and Politics in Western Europe, Bristol, Frank Cass, 1985, p. 19-37; Sandro Bellassai, La morale comunista : pubblico e prin vato nellit rappresentazione del PCI (1947-1956), Rome, Carocci, 2000

(3) Sylvie Chaperon, « Lingresso delle donne nella vita política : Francia e Italia a confronto $»$, Genesis, 5 (2), 2006, p. $117-136$. 
et il se trouve confronté à un mouvement féministe radical très puissant et en même temps peu intéressé par la politique au sens habitue du terme ${ }^{l}$.

Mónica Moreno-Seco,

Uriversité d'Alicante, Département de sciences bumaines, 03080, Alicante, Espagne.
Mâtresse de contérences en histoire contemporaine à l'Université d'Alicante, spécialiste en histoire des femmes et en histoire du genre, Mónica Moreno-Seco est l'auteur de Manifiestos feministas : antología de textos del movimiento feminista español (Université d'Alicante, 2005) et de divers articles parus dans les revues History and Anthropology, Ayer, Historia y Polltica, Historia Contemporánea et Memoria e Ricerca. (monicamoreno@ua.es)
(1) Dominique Loiseau, « La politique du PCF et les femmes depuis $1945 \%$, in Christine Delphy et Sylvie Chaperon, Cinquantenaire du Deuxxieme sexe, Paris, Syllepse, 2002, p. 387-391. Nous remercions Michel Christian pour l

\section{Note aux contributeurs}

\section{Articles}

Les manuscrits, inéstits et non sroumis paral. leventent a une autre revue, seront adresse-

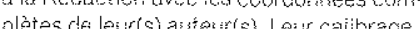
sera d'environ 40000 sionos (7000 mats) espaces et notes comprises.

\section{Conventions}

Les références bab biliographiquies sont inteGrees dans les notes de bas de page t:les sont presentées de la maniere suivante po: holviage : Prenom. Nom, mre de louvrege Prénom Nom, sTitre de l'astichen Nom de a revuse numéro, date parges : pour line contribution à un ouvrage collectif: Prénon Nom, «Tisre de la contribution», in Prên Nom du directevtr of lourrage, Titre de rouvrage, Vhe, Ed our, annee, pages. Sise (tableas: veillera à leur piace, ráference ch appel da le texte, ainsi qu'à la mention systématiquie des titres, iegendes, sources et tềtières. pas héesiter à contacter ia Réstlaction.
Procédure

Les articles pris en considération par la

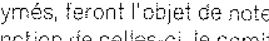
de Rédaction prend l'une des trois décisior suivantes: acceptation en l'état, refiss. acceptation sous risenve do modncato maximal de quatre mois après réception du manuscrit.

En cas d'acceptation, il sera joint à la demière version du texte : a) un résumé de 150 signes t 15 motsi, a ccompagne de sa tha duchen en anglars, b) cha mols cles, c) une

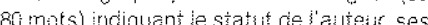
derrières publications et son adresse sioc tronique. Unie fois acceptés, ies textes font eventuelement lobjet d un thavalis editorial. Le ilibelie final des titres et le chapeau des

Rubriques

Si vous scuhaitez contribuer aux rubricues de la reyue, nous vous invitons à contacter

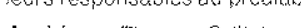

Archives : Thomas Griliot (thomas.grillo@grmail.com) et Gilles Morin
Avis de recherches : Quentin Deltuermoz n.deivermoz@gmailicomi et Mar imariebvincertigyaho.f

Images, lettres et Sons : Ludivine Bantigny (iudivingabantigny@free.sr) Vincent Lemire

(Vemireyncent@gmail.com)

Vingtième Siècle signale : Johann Charoutot johannchapouto@onotnail. com), xavler vigna

gne fr) et Claire

List faure vustine Faur

Marie Scot (marieschet@sciences-po.fr) et Charlotte Vorms fcyorns@gmail.com)

Ungtième Siècle. Revise d'histoire est ndexée notamment danis les bases do AISPRPSA, Ametica: History and Lifo Arts \& Humanities Citation Index, Franc Historical Abstracts, iBSS-intemational Bibllography of the Social Sciences. IBZ. international Bioliography Apts Humanities Periosical Cont PA.S 


\section{Vingtième Siècle. Revue d'histoire} $n^{0} 126$ avril-juin 2015

\section{DOSSIER}

\section{Femmes, genres et communismes}

Michel Christian et Alix Heiniger

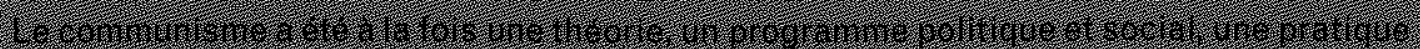

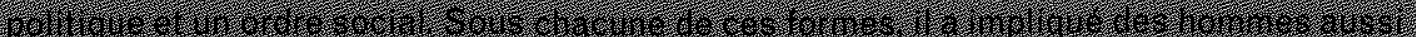

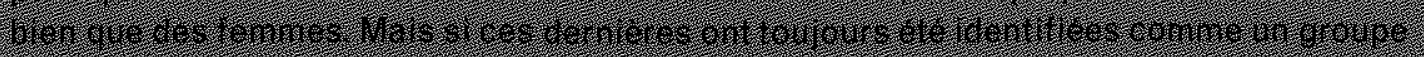

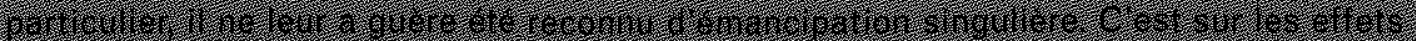

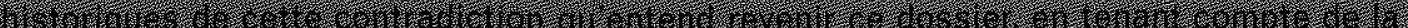

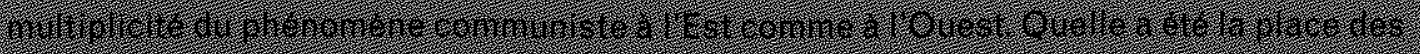

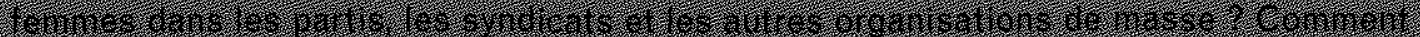

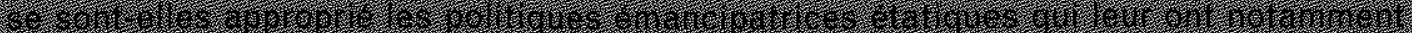

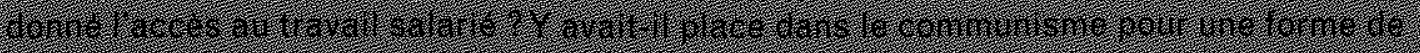
teminisine?

\section{Rabilowes}

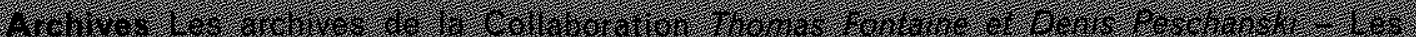

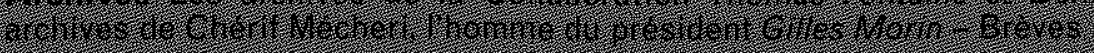

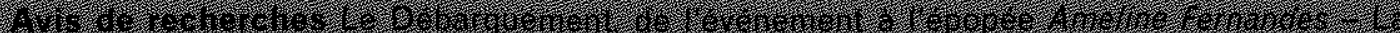

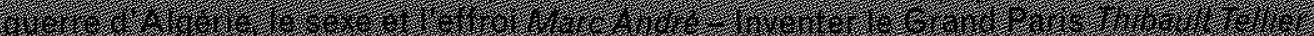

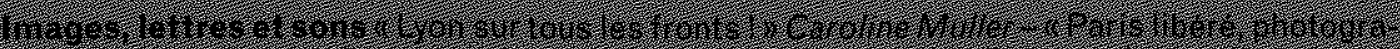

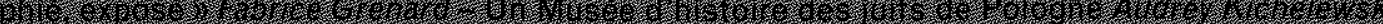

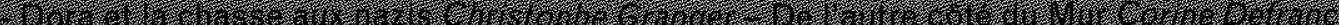

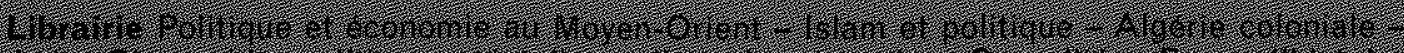

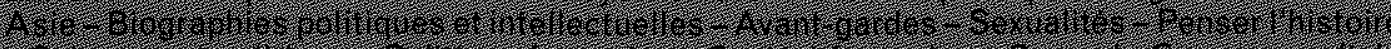

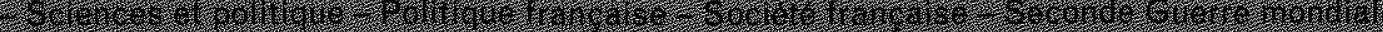

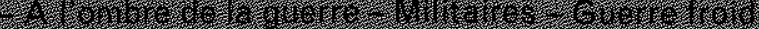

\section{Presses de Sciences Po}

117, boulevard Saint-Germain - 75006 Paris - France

Tét : + 33 (0)1 45498364 - Fax : +33 (0)1 45498334 - Diffusion/distribution CDE/SODIS

http: //www.pressesdesciencespo.fr

Retrouvez la revue sur http:///www, cairn. info et hitp:i//www.persee.ft

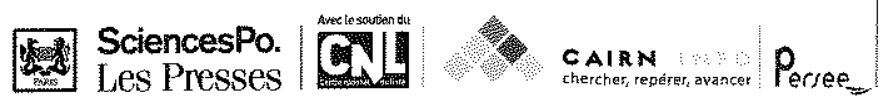

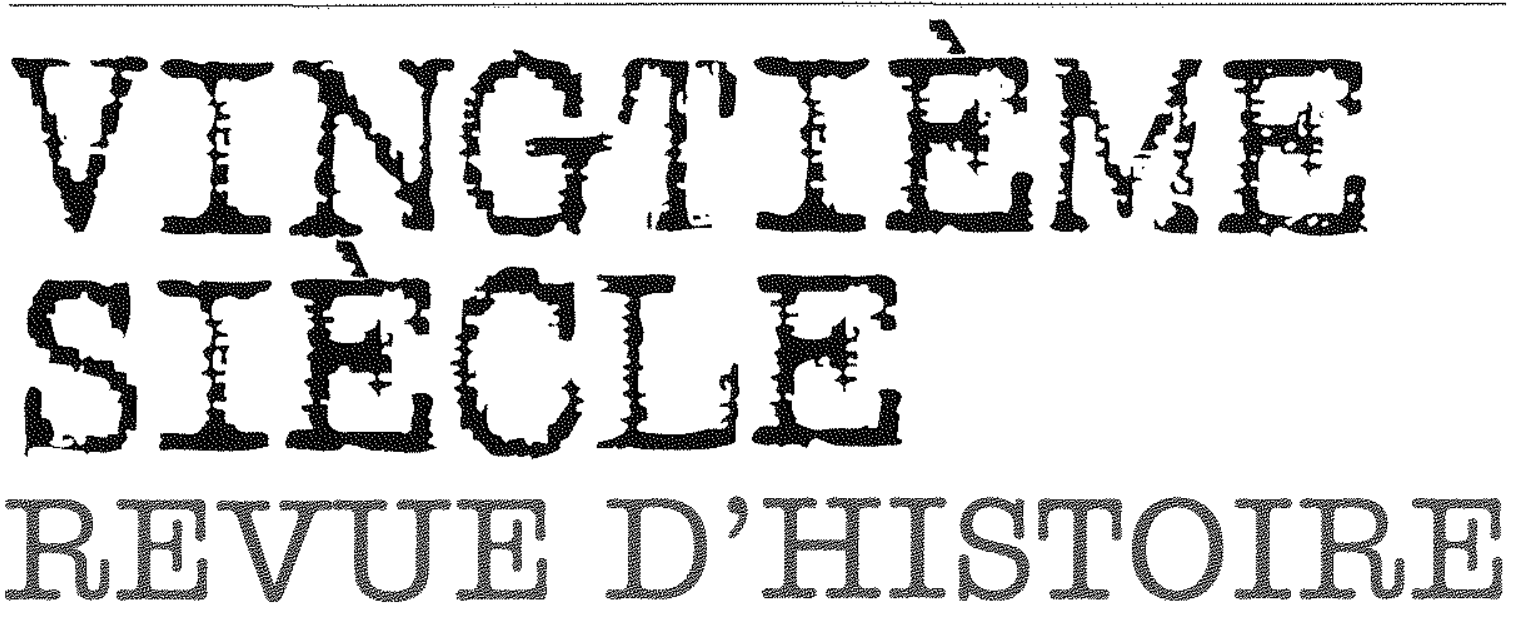

Numéro 126 | avril-juin 2015

\section{DOSSIER}

Femmes, genres et communismes Michel Christian et Alix Heiniger

Malgor tata Fidelis Femmes el communisme en Europe de IEst Thong Tang Lir emarcipation destemmes s en Chine Nadege Ragaruldenties de genre et theatre en Bulgarie Magal Eelalovo Les temmes du Krenila

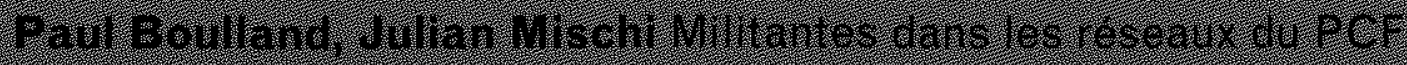
Whal elmistan Eenmes et hommes at sein do SED HIX Weinger Les comnunistes allemands au pusme du genre Celra borret Femmes, communisme et internationalisme

Monica Moreno-seco parl communisie et téminisme en Espaente

Tres Denechere tes paranages dentants etrangers

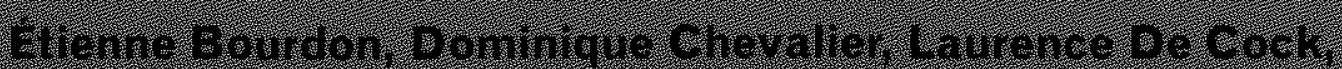
ahristan Delacroix, Patrich Carcia, Jean Ledic. Le CAPES dhistare et de geographie. 